\title{
Photonic band gap effect and structural color from silver nanoparticle gelatin emulsion
}

\author{
Mang Hin Kok, Rui Ma, Jeffrey Chi Wai Lee, Wing Yim Tam, ${ }^{*}$ C. T. Chan, and Ping Sheng \\ Department of Physics and Institute of Nano Science and Technology, Hong Kong University of Science and Technology, Clear Water \\ Bay, Kowloon, Hong Kong, China \\ Kok Wai Cheah \\ Department of Physics, Centre for Advanced Luminescence Materials, Hong Kong Baptist University, Kowloon Tong, Hong Kong, China
}

(Received 22 June 2005; published 21 October 2005)

\begin{abstract}
We have fabricated planar structures of silver nanoparticles in monochromatic gelatin emulsion with a continuous spacing ranging from $0.15-0.40$ micron using a two-beam interference of a single laser source. Our planar holograms display a colorful "rainbow" pattern and photonic bandgaps covering the visible and IR ranges. We model the planar silver nanoparticle-gelatin composite system using an effective medium approach and good agreement is obtained between theory and experiment.
\end{abstract}

DOI: 10.1103/PhysRevE.72.047601

PACS number(s): 42.40.-i, 42.70.Qs, 78.67.Bf

Holographic lithography, a method combining the well developed techniques of holography and photoinduced polymerization, has been widely used recently to fabricate microstructures [1]. One potential application for these structures is in photonic crystal in which EM wave propagation is forbidden within a band of wavelengths, analogous to band gaps in semiconductors [2]. The holographic lithography, because of its flexibility in achieving various crystal structures using multibeam interference, has some advantage over the conventional techniques of self-assembly of colloidal microspheres and microfabrication in fabricating photonic crystals [3]. However, common holographic lithography is limited to the use of polymeric material that has relatively low dielectric constant, hence hindering the fabrication of a complete photonic band gap. Increasing the dielectric contrast is thus highly desirable, and various attempts such as infiltrations of high dielectric materials, e.g., metals, into self-assembly microspheres have been pursued with some success [4]. It is known that $\mathrm{Ag}$ is used as the recording material in common photography as well as in reflection hologram where images are stored as Ag nanoparticles embedded in a gelatin. The $\mathrm{Ag}$ particles provide the high dielectric contrast needed for photonic band gap materials. It has also been demonstrated recently that several newly developed silver halide-sensitized gelatin emulsions can provide the needed resolution, more than 5000 line $/ \mathrm{mm}$, for visible range applications [5]. In particular, planar as well as 3D structures can be fabricated from these emulsions using similar setups for the holographic lithography $[5,6]$. Moreover, a multiple band reflection hologram has been fabricated using new panchromatic emulsions by multiplexing three laser sources with the Denisyuk configuration in which a normal incident beam, after passing through the emulsion, is reflected back from a mirror behind the holographic plate to create planar structure inside the emulsion $[5,7]$. In a 1D planar hologram, the spacing of the planar structure depends on the wavelength of the light

*Corresponding author. FAX: 852-2358-1652. Email address: phtam@ust.hk source used. However, the spacing can also be varied by controlling the incident angle of the beam. Here, we report the use of a two-beam interference to fabricate planar structure of silver nanoparticles with various spacings in monochromatic emulsion using a single laser source. Our planar holograms, having spacing ranging from 0.15 to $0.40 \mu \mathrm{m}$, show bright reflections and band gaps covering the visible and IR ranges. Furthermore, we are able to fabricate a hologram with a continuous planar spacing on a holographic plate by continuously varying the incident angle of the laser beam in one single exposure, and thus creating a colorful "rainbow" pattern on the plate. We have also calculated the optical properties of the planar silver nanoparticle-gelatin composite and good agreement is obtained between theory and experiment.

Figure 1(a) shows the schematic of the setup for the twobeam interference experiment. A laser beam from an argon ion laser (Innova 70-5, Coherent), after passing through a

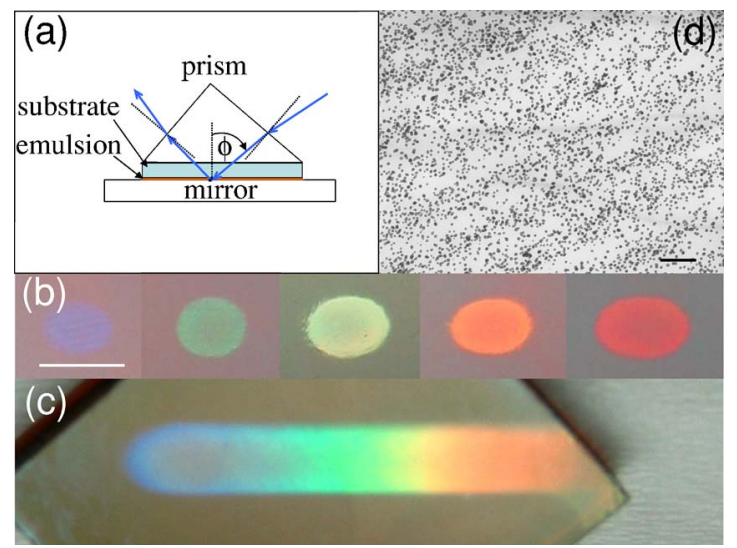

FIG. 1. (Color online) (a) Schematic setup for the two-beam interference. (b) Samples fabricated at incident angles $\phi$; from left to right: $0^{\circ}, 29^{\circ}, 38^{\circ}, 43^{\circ}$, and $47^{\circ}$. The scale bar is $4 \mathrm{~mm}$. (c) Sample fabricated with a continuous incident angle from $0^{\circ}$ (left) to about $45^{\circ}$ (right). The spread of the rainbow is about $18 \mathrm{~mm}$ long. (d) TEM image of the cross section of the sample developed by D19 at $43^{\circ}$ incident angle. The scale bar is $200 \mathrm{~nm}$. 
prism, enters from the glass substrate side of the holographic plate and interferes inside the emulsion with its reflection from a mirror behind the emulsion. The interference pattern consists of planes parallel to the substrate surface with spacing $d$ given by

$$
d=\frac{\lambda}{2 n \cos \phi},
$$

where $\lambda(488 \mathrm{~nm})$ is the wavelength of the laser source, $n$ (1.59) is the refractive index of the gelatin emulsion [8], and $\phi$ is the incident angle inside the emulsion. We used the 488 $\mathrm{nm}$ line of the argon ion laser, beam size $4 \mathrm{~mm}$, power 1.1 $\mathrm{mW}$, and polarization perpendicular to the plane formed by incident and reflected beams, for our holographic setup. The prism, refractive index 1.52 (the same refractive index as the glass substrate of the holographic plate), was chosen such that incident angles larger than the critical angle $\left(\phi_{c}=39^{\circ}\right)$ for the emulsion can be achieved. In our experiment we used two prisms and internal angles $30^{\circ} / 60^{\circ} / 90^{\circ}$ and $45^{\circ} / 45^{\circ} / 90^{\circ}$ to cover incident angles $\phi$ of $0^{\circ}-67^{\circ}$, corresponding to spacings of $0.15-0.40 \mu \mathrm{m}$. Index matching fluids were applied to the prism-substrate and emulsion-mirror interfaces to eliminate multiple reflections. The whole experimental setup was mounted on a motorized rotating stage such that the incident angle could be varied continuously. An ultra-fine-grain monochromatic emulsion PFG-03M (Slavich, Russia), around $6 \mu \mathrm{m}$ thick and resolution much better than $5000 \mathrm{l} / \mathrm{mm}$, on glass substrate was used for our experiment. After exposure with optimal time $1.5 \mathrm{~s}$, the emulsion was developed using GP2 developer as recommended by the manufacturer to produce the planar structure of the $\mathrm{Ag}$ nanoparticle-gelatin composite [9]. Figure 1(b) shows pictures (taken under white light) of samples with different spacing fabricated in the visible range. The samples show, from left to right, blue, green, yellow, orange, and red colors, fabricated using incident angles $0^{\circ}, 29^{\circ}, 38^{\circ}, 43^{\circ}$, and $47^{\circ}$, respectively. The samples are relatively uniform with size as large as the beam diameter. Figure 1(c) shows a picture of a sample with continuous spacing displacing a "rainbow" pattern, from blue to orange-red, in the visible range. This sample was obtained by continuously changing the incident angle, from $0^{\circ}$ to about $45^{\circ}$, in one single exposure. The axis of rotation and the rate were carefully adjusted such that the interference beams swept across the holographic plate with the right exposure time. Figure 1(d) shows the cross section TEM image of a sample fabricated using PFG-03M emulsion on triacetate substrate with incident angle $\phi=43^{\circ}$ and developed using another developer D19 [10]. The D19 developer, though with slightly less resolution compared to GP2, was used because it gave harder samples for ultramicrotomy to obtain $80 \mathrm{~nm}$ thick slices for TEM imaging. It is not difficult to see from Fig. 1(d) that the Ag particles, mean size 15.5 $\mathrm{nm}$, form planar structure with spacing $\sim 0.25 \mu \mathrm{m}$ [11]. The volume fraction of the $\mathrm{Ag}$ particles was found to have a sinusoidal variation with a mean of $2.3 \%$ and a SD of $0.8 \%$. To obtain the reflectance and transmittance of the sample, light from a $150 \mathrm{~W}$ halogen lamp was collimated into a beam with diameter $\sim 0.5 \mathrm{~mm}$ and shone onto the sample from the

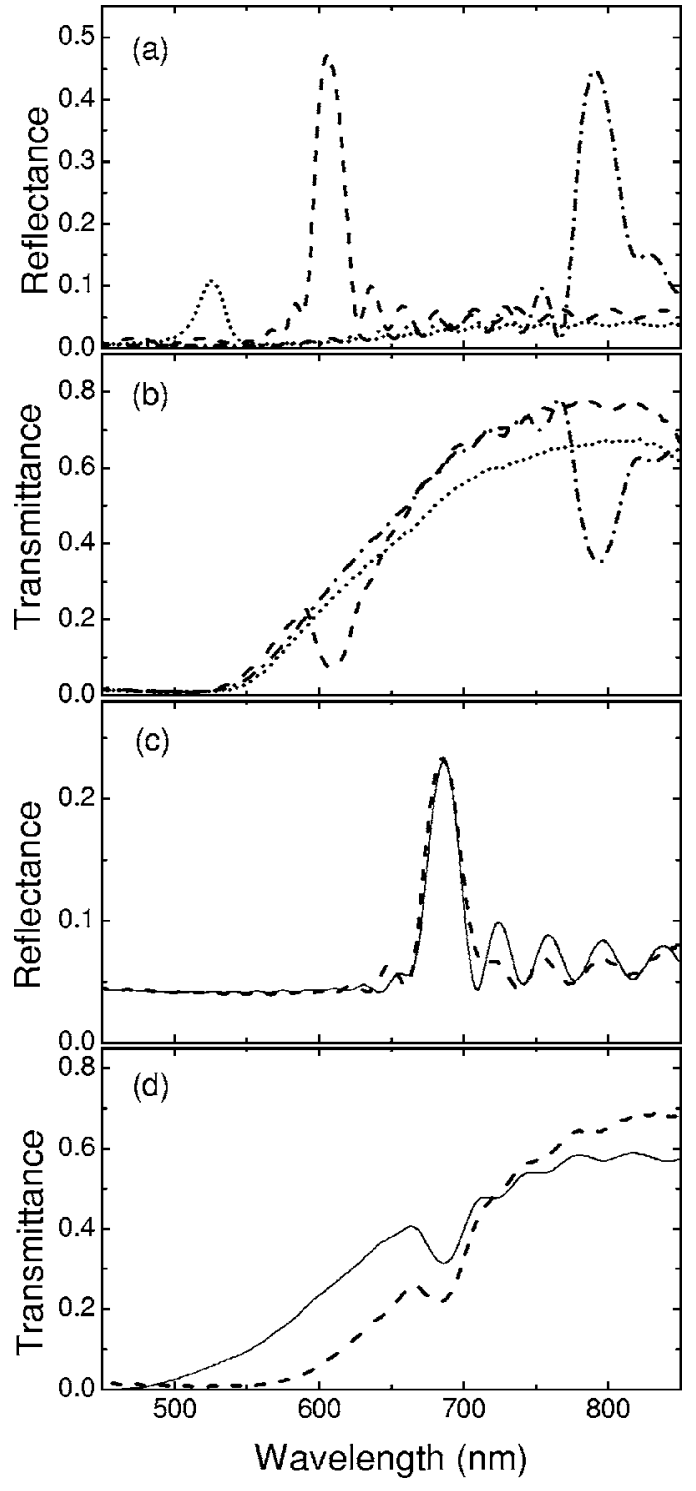

FIG. 2. (a) and (b): Normal reflectance and transmittance for samples developed using GP2 with $\phi=29^{\circ}$ (dotted line), $43^{\circ}$ (dashed line), and $56^{\circ}$ (dot-dash line); (c) and (d) for sample (dashed line) with $\phi=43^{\circ}$ corresponding to Fig. 1(d) using D19 developer and model calculation (solid line). In (c) the reflectance from the glass substrate has been added to the sample reflectance for comparison with the model calculation.

substrate side. Furthermore, a wedged glass was attached to the substrate with index matching fluid in between such that the reflection projected on a screen from the sample could be separated from that of the substrate reflection. This enabled measuring the sample reflection directly, avoiding the substrate reflection. An optical fiber was used to couple the reflected light to a spectrometer (Oriel Cornerstone TM260) for spectral analysis. The optical setup allowed almost normal incident reflection and transmission measurements. Figure 2 shows the reflectance and transmittance for three samples with $\phi=29^{\circ}, 43^{\circ}$, and $56^{\circ}$. Observed directional band gaps are consistent with the reflection pictures of Fig. 1(b). Note that in addition to the band gaps, there are side oscillations due to the Fabry-Pérot effect of the thin emulsion 


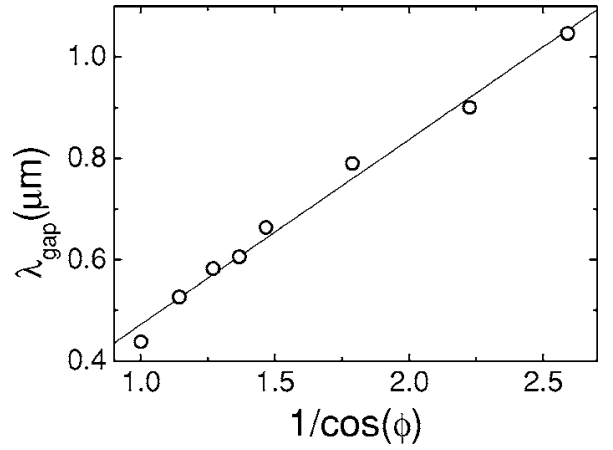
GP2.

FIG. 3. $\lambda_{\text {gap }}$ versus $1 / \cos \phi$ for all samples developed using

film. The reflectance is large at long bandgap wavelength $\left(\lambda_{\text {gap }}\right)$ while it is small for wavelength below $550 \mathrm{~nm}$. This is due primarily to the strong absorption (including that of the Ag nanoparticles) of the emulsion at wavelength less than $550 \mathrm{~nm}$ as shown in the transmittance measurements. The absorption can be significantly reduced by bleaching the sample, transforming Ag to silver bromide. However, the band gaps would shift slightly due to the bleaching effect. The reflectance and transmittance corresponding to the sample developed with D19 in Fig. 1(d) are shown in Figs. 2(c) and 2(d), respectively. Note that the band gap is redshifted by about $13 \%$ as compared to the sample in Fig. 2(a) with the same incident angle but developed using GP2. Plots of the band gap wavelengths, ranging from 0.44 to $1.04 \mu \mathrm{m}$, are shown in Fig. 3 as a function of $1 / \cos \phi$ (open circles) for all the samples developed using GP2. It is clear that $\lambda_{\text {gap }}$ is inversely related to the cosine of the incident angle as indicated by the straight line, consistent with $\lambda_{\text {gap }} \sim d$ of Eq. (1).

We model the Ag nanoparticle-gelatin system as a layered structure of Ag gelatin on a $2 \mathrm{~mm}$ thick glass substrate with spatial dielectric constant given by an effective medium approach obtained from the Maxwell-Garrent formula [12]. The dielectric function of the Ag nanoparticles is obtained from a modified Drude model [13] which takes the form

$$
\varepsilon(\omega)=\varepsilon_{a}-\frac{\left(\varepsilon_{b}-\varepsilon_{a}\right) \omega_{p}^{2}}{\omega(\omega+i \gamma)},
$$

where $\varepsilon_{a}=5.45, \varepsilon_{b}=6.18, \omega_{p}=1.72 \times 10^{16} \mathrm{rad} / \mathrm{s}$, and the absorption term $\gamma$ is given by

$$
\gamma=\frac{v_{F}}{l}+\frac{v_{F}}{r},
$$

for $v_{F}=1.38 \times 10^{6} \mathrm{~m} / \mathrm{s}$ (Fermi velocity), $l=52 \mathrm{~nm}$ (electron mean free path at room temperature), and $r=d / 2$ with $d$ be- ing the diameter of the Ag particle $[13,14]$. Equation (3) is valid for particle size around $10 \mathrm{~nm}$. It is found that the emulsion itself has rather strong absorption below $550 \mathrm{~nm}$; an absorption term obtained from fitting the reflectance and transmittance from developed unexposed emulsion is thus added to the gelatin. The dotted lines in Figs. 2(c) and 2(d) show the reflection and transmission spectra calculated by the scattering matrix method (SMM) [15] for a layered structure corresponding to Fig. 1(d). We have used a sinusoidal variation, from $0.6 \%$ to $4.0 \%$, in the volume fraction of $\mathrm{Ag}$ nanoparticles and a period of $210 \mathrm{~nm}$ given by Eq. (1) to obtain the best fit to the data. Furthermore, the scattering matrix is constructed by dividing a single period into 64 layers with the dielectric function taken to be constant within each sublayer. There is no significant change in the result by further increasing the number of discrete sublayers used in the calculation. The Ag nanoparticles are taken to have a size of $15 \mathrm{~nm}$ in diameter. It is found that the particle size affects only the absorption magnitude but not the band gap position. The effective thickness of the gelatin is adjusted to be $4.83 \mu \mathrm{m}$ by matching the Fabry-Pérot oscillations in Fig. 2(c). It is clear from Figs 2(c) and 2(d) that the reflection and transmission peaks match very well with the experimental results. Moreover, the overall agreement of the spectra and the Fabry-Pérot peaks is much better than satisfactory, indicating that the model works well for our system. Note that good quality fits to the GP2 samples can also be obtained when the spacing of the layer structures, the volume fraction, and the particle size are treated as free fitting parameters.

To conclude, we have fabricated planar structure consisting of $15 \mathrm{~nm} \mathrm{Ag}$ particles embedded in monochromatic holographic emulsion by using a two-beam interference from a single light source. Furthermore, a sample with continuous spacing, showing a colorful "rainbow" pattern, is fabricated on a single plate by continuously changing the incident angle during one single exposure. Bandgaps covering the visible and IR ranges are observed showing a linear relation with the spacing of the samples. Good agreement with experiment is obtained by modeling the Ag nanoparticle-gelatin system with a planar structure using an effective medium approach. Lastly, the current work can be easily extended to fabricate 2D/3D structures of the Ag nanoparticles composite using multibeam configurations, leading to new prospects.

Support from Hong Kong RGC Grants No. CA02/ 03.SC01 and No. HKUST603303 is gratefully acknowledged. We thank C. H. Wong and H. W. Tsang for technical help.
[1] M. Campbell et al., Nature 404, 53 (2000); S. Yang et al., Chem. Mater. 14, 2831 (2002); Y. V. Miklyaev et al., Appl. Phys. Lett. 82, 1284 (2003); X. Wang et al., Appl. Phys. Lett. 82, 2212 (2003); X. Wang et al., Adv. Mater. (Weinheim, Ger.) 15, 1526 (2003).
[2] See, e.g., Photonic Band Gaps and Localization, edited by C. M. Soukoulis (Plenum, New York, 1993); C. M. Soukoulis, J. Opt. Soc. Am. B 10, 208 (1993); Photonic Band Gap Materials, edited by C. M. Soukoulis (Kluwer, Dordrecht, 1996); E. Yablonovitch, Phys. Rev. Lett. 58, 2059 (2003); S. John, Phys. 
Rev. Lett. 58, 2486 (1987); Photonic Crystals, edited by J. D. Joannopoulos, R. D. Meade, and J. Winn, (Princeton University Press, Princeton, NJ, 1995).

[3] See, e.g., C. C. Cheng et al., Phys. Scr., T T68, 17 (1996); S. Noda and A. Sasaki, Jpn. J. Appl. Phys., Part 1 36, 1907 (1997); S. Y. Lin et al., Nature 394, 251 (1998); A. van Blaaderen, Science 282, 887 (1998).

[4] See, e.g., J. E. G. J. Wijnhoven and W. L. Vos, Science 281, 802 (1998); E. Palacious-Lidon et al., Appl. Phys. Lett. 81, 4925 (2002);W. Y. Zhang et al., Phys. Rev. Lett. 84, 2853 (2000); Y. Jiang et al., J. Phys.: Condens. Matter 15, 5871 (2003); W. Li et al., J. Phys.: Condens. Matter 17, 2177 (2005).

[5] J. M. Kim et al., Appl. Opt. 40, 622 (2001); 41, 1522 (2002).

[6] Z. Ye et al., Phys. Lett. A 299, 313 (2002); G. P. Wang et al., J. Mod. Opt. 50, 2155 (2003); M. Ulibarrena et al., Proc. SPIE 5450, 76 (2004).

[7] M. Ulibarrena et al., Opt. Express 22, 3385 (2003).

[8] Our measured refractive index for the gelatin is close to that reported in A. Belendez, T. Belendez, C. Neipp, and I. Pascual, Appl. Opt. 41, 6802 (2002).

[9] The developing chemicals and procedures for GP2 are listed in the report "Technical product specifications and sales information brochure," Geola, 2001. The GP2 is a solution-physical process developer that gives high resolution because of finer $\mathrm{Ag}$ particles. However, it gives too soft a sample, possibly due to formation of microvoids inside the gelatin (see Ref. 5), for getting good TEM cross sectional images because of serious deformations during the TEM preparation processes.

[10] The developing chemicals and procedures for D19 can be found in Silver Halide Recording Materials for Holography and Their Processing, edited by H. I. Bjelkhagen, Springer Series in Optical Sciences Vol. 66 (Springer-Verlag, Heidelberg, New York, 1993). The D19 is a chemical process developer that gives larger Ag particles with less resolution compared to the GP2 developer. However, it gives harder samples for cross section TEM imaging.

[11] Note that the spacing of Ag layers could change due to the deformation from the ultra-microtomy process. The particle size and the spacing are obtained from averaging over a larger image of the cross section consisting of $\sim 2000$ particles.

[12] See, e.g., T. C. Choy, Effective Medium Theory : Principles and Applications (Oxford University Press, Oxford, 1999).

[13] J. J. Xiao, J. P. Huang, and K. W. Yu, Phys. Rev. B 71, 045404 (2005); P. G. Kik, S. A. Maier, and H. A. Atwater, Phys. Rev. B 69, 045418 (2004).

[14] D. P. Peters, C. Strohhöfer, M. L. Brongersma, J. van der Elsken, and A. Polman, Nucl. Instrum. Methods Phys. Res. B 168, 237 (2000).

[15] N. Stefanou, V. Yannopapas, and A. Modinos, Comput. Phys. Commun. 113, 49 (1998); N. Stefanou, V. Yannopapas, and A. Modinos, Comput. Phys. Commun. 132, 189 (2000). 